\title{
Análise da Utilização de Interface Multimodal para Aplicação de TV Digital e Segunda Tela
}

\author{
Renan Patrick de C. Marçal, Cláudia Caroline L. de Oliveira, Marcos César da \\ Rocha Seruffo
}

Laboratório de Desenvolvimento de Sistemas (LADES) - Faculdade de Computação -

Universidade Federal do Pará (UFPA) - Campus Castanhal

Caixa Postal 15.064 - 91.501-970 - Castanhal - Pará - Brasil

renancarvlho@gmail.com, carollemarc@hotmail.com, seruffo@ufpa.br

Abstract. This paper aims to conduct a comparative use of natural user interfaces, through the use of an interactive application that streamlines the interrelationship between the user and the digital TV, using for this second screen and the kinect device. The evaluation of user experience quality is performed through quantitative and qualitative metrics. The purpose of this proposal is to find the means of more direct and natural interaction goal to the user's needs, so it does not interfere with the traditional way of watching TV.

Resumo. Este artigo tem como objetivo realizar um comparativo da utilização de interfaces naturais do usuário, através do uso de uma aplicação interativa que dinamiza a inter-relação entre o usuário e a TV digital, utilizando para isso segunda tela e o dispositivo kinect. A avaliação da qualidade de experiência do usuário é realizada através de métricas quantitativas e qualitativas. $O$ objetivo desta proposta é encontrar o meio de interação natural mais direto e objetivo ás necessidades do usuário, de forma que não interfira na maneira tradicional de assistir TV.

\section{Introdução}

A interação homem-computador (IHC) tem sido um dos grandes focos dos desenvolvedores nos últimos tempos. Estudos relacionados à qualidade de experiência do usuário, seu nível de satisfação e facilidade de interação com o sistema, tem ganhado destaque em congressos, pesquisas e afins. Segundo [Karray 2008] o conceito de IHC formou-se juntamente com o surgimento dos computadores e mais recentemente das máquinas em geral, devido ao fato de que algumas máquinas precisam ser utilizadas de forma adequada pelo homem.

Com o crescimento de estudos das interfaces na área de IHC e o grau de influência que estas exercem sobre a interação com o usuário, desenvolveu-se este trabalhado visando avaliar e medir o nível de interação do usuário através de Interfaces Naturais (INs). Optou-se por abordar a área de TV Digital Interativa (TVDi), devido sua consolidação e crescente ascensão no cenário atual da rede de televisão brasileira.

Segundo um estudo do IBOPE, realizado em 2014, encomendado pela Secretária de Comunicação Social (SECOM), 97\% dos entrevistados veem TV enquanto que 47\% têm o hábito de acessar a internet, porém, os valores diferem um pouco quando se trata de preferência, segundo o mesmo estudo, a TV é o meio preferido para $76,4 \%$ da população, enquanto que 13,1\% preferem a internet. [IBOPE, 2014]

Em conjunto com a TVDi foi feito uso de segunda tela, conceito este que atualmente está ganhando maior visibilidade e interesse na área acadêmica, aumentando 
o número de pesquisas e aplicações em desenvolvimento. A segunda tela ou second screen é utilizada como complemento da interação por INs, acarretando em uma interação híbrida por parte do usuário, através da disponibilização de conteúdo adicional.

Através da avaliação da utilização da interface multimodal, este trabalho visa uma comparação das interfaces naturais por gestos e por voz, por meio de métricas quantitativas e qualitativas. Observando a qualidade de interação e o grau de satisfação dos usuários.

Este artigo está dividido em sete seções. Na segunda seção é feita a revisão literária descrevendo de forma sucinta as tecnologias e conceitos que serviram de base para este trabalho. $\mathrm{Na}$ terceira seção são citados os trabalhos que serviram de fundamento para o desenvolvimento deste estudo. Na quarta seção é mostrada a arquitetura desenvolvida, onde é explicado o funcionamento da aplicação desenvolvida, algumas etapas do desenvolvimento. $\mathrm{Na}$ quinta seção é descrito o estudo de caso, o ambiente de testes utilizado e a forma como ocorreram os testes. Na sexta seção são citados os resultados obtidos pelos testes e questionários realizados com usuários. Por ultimo a sétima seção é composta pela conclusão, relatando as considerações finais e as propostas de trabalhos futuros.

\section{Revisão literária}

Nesta seção serão abordados (de forma sucinta) as principais tecnologias e conceitos que foram utilizados para o desenvolvimento do aplicativo proposto neste artigo, pois, durante a aplicação do questionário na fase de testes, notou-se falta de conhecimento dos usuários sobre estes conceitos.

\subsection{Interface Natural do Usuário}

O papel básico da interface do usuário é permitir que se estabeleça a comunicação entre a máquina e o usuário. Quanto mais simples de manusear e menor o grau de dificuldade para aprender a manipular determinada interface, maior será a capacidade de interação humano-computador. "De fato, a solução perfeita seria aquela na qual o usuário nem percebesse a utilização de uma interface" [Tebbutt 1995].

A evolução das interfaces, associada à busca pela melhor qualidade e facilidade de interação do usuário com sistemas computacionais, caminha cada vez mais para a utilização de interfaces naturais (Natural User Interface - NUI), como a opção mais intuitiva e direta dentre as alternativas existentes. NUI segue um novo paradigma com o objetivo de respeitar a percepção humana. Segundo [Valli 2007], interagir com sistemas com Interfaces Naturais é extremamente intuitivo e agrada a todos envolvidos.

\subsection{TV Digital Interativa}

Duas outras tecnologias, abordadas no presente trabalho, ascendentes no campo dos desenvolvedores e pesquisadores da ciência da computação, são a TVDi (TVDi) e a segunda tela, tecnologias que também visam o conforto dos usuários. A característica básica das iTVs é a possibilidade de controle do usuário sobre a experiência com a TV, podendo definir o curso de interação com o conteúdo disponibilizado. [EY 2013]

Segundo [Fernandes 2004, p. 22], "Um sistema de televisão digital interativa deve adotar e integrar um conjunto de diferentes tecnologias de hardware e software para implementar suas funcionalidades. Os conteúdos de interatividade apresentados 
durante a programação da TV podem ser exibidos na primeira tela (geralmente a TV), no menu desta ou através de uma Segunda Tela.

\subsection{Segunda tela}

O uso da internet aliado a conteúdos televisionados tem modificado as formas tradicionais de o usuário assistir TV, enquanto recebe informações da primeira tela o telespectador pode compartilhar e trocar informações com outros usuários através da segunda tela. É definido como segunda tela a ação de utilizar dispositivos móveis como meio de interação para compartilhamento e troca de informações com outros usuários enquanto assiste a programas de TV

A utilização de segunda tela é realizada através do acesso ao conteúdo adicional da programação televisionada na primeira tela. Para a entidade que dirige e que rege todas as ações e estratégias das empresas referentes a segunda tela, essa, é toda e qualquer "experiência de engajamento da audiência, que inclui a TV Social como um elemento integrante". [SET, 2013].

Utilizou-se a aplicação (app) Dengue Fever (DFapp) para acesso do conteúdo adicional. O DFapp é um app que tem o foco na doença Dengue, uma doença que mata milhares de pessoas por ano no Brasil. Através da utilização deste app são disponibilizadas informações adicionais sobre a doença em questão.

Neste trabalho foi feito uso de autenticação através do uso do QR Code, por sua velocidade de processamento e sua interação com dispositivos móveis (segunda tela). $\mathrm{O}$ termo QR é acrônimo de Quick Response, que em português significa resposta rápida. É um código de barras 2D que pode ser detectado e traduzido por celulares que possuem uma câmera e um aplicativo específico para leitura deste código [Gazolla neto 2012]

\section{Trabalhos Correlatos}

As tecnologias abordadas nesta pesquisa estão sendo estudadas há algum tempo e têm ganhado destaque no âmbito das Tecnologias de informação e comunicação. Vários trabalhos se destacam nessa área, embora a maioria não una essas tecnologias em um trabalho apenas.

[Cruickshank 2007] desenvolveu um trabalho de pesquisa em que este analisou a qualidade da interação dos usuários com a TVDi e as respectivas barreiras encontradas na utilização das correntes interfaces de interação utilizadas, através disso, propôs uma solução para a problemática adicionando o uso de segunda tela, durante a utilização das TVDi's, diminuindo o número de informações na primeira tela e melhorando a interação dos usuários com a TV e a navegação pelos serviços disponíveis.

[Turk 2013] realizou uma análise sobre a interação multimodal, passando através da evolução, das possibilidades e dos desafios da área, focando em um dos pontos críticos no que se refere a interação multimodal; a integração dos diferentes modos. O autor pondera sobre as possibilidades de interação precoce ou tardia, analisando evidências dos diferentes tipos.

[Kong 2011] quantifica a preferência dos usuários por cada modalidade de interação (interface multimodal), e busca adaptar o modelo de interação baseado em requisitos de QoS analisados. Através da realização de testes, compara as modalidades de interação selecionadas automaticamente e as selecionadas aleatoriamente, obtendo resultados satisfatórios para a modalidade de interação selecionada automaticamente, baseada nos ajustes feitos através da análise das necessidades pessoais dos usuários. 
[Bobeth 2014] realizou um trabalho semelhante ao proposto neste artigo, analisando a qualidade de interação em plataformas multimídias de TVDi de diferentes aplicações populares na área. $O$ autor analisa três diferentes tipos de entrada de interação com a TV Digital (tablet, gesto mão-livre, controle remoto), analisando o efeito da performance em diferentes grupos etários, e a influência da idade na qualidade de experiência dos usuários. Através do estudo constatou grande influência da modalidade de dispositivo de entrada sobre a performance e qualidade de experiência, enquanto que a idade pareceu não influenciar na mesma.

A diferença deste trabalho para [Bobeth 2014], é que no presente trabalho são avaliada duas formas de NUI (voz e gesto) levando em consideração a qualidade da experiência do usuário, quanto que Bobeth avalia diferentes tipos de interfaces levando em consideração a influencia da idade dos usuários na qualidade de experiência dos diferentes métodos interativos.

Utilizou-se TV Digital e a tecnologia de segunda tela em conjunto com o uso de interfaces focando o estudo assim como [Bobeth 2014], na análise dos dispositivos de entrada, usando porém, comandos por gestos e por voz. É realizada ainda uma análise do perfil dos usuários através da mineração dos dados coletados por meio de testes, procurando delimitar fatores importantes que influenciam a performance e a qualidade de experiência dos usuários.

\section{Arquitetura Desenvolvida}

A arquitetura da aplicação proposta foi desenvolvida com o intuito de dar ao usuário da TVDi total liberdade de interação, sem afetar a usabilidade das funções padrões da TV ou prejudicar os comportamentos tradicionais dos usuários que assistem televisão.

A utilização de recursos de segunda tela através da aplicação desenvolvida pode ser feita a qualquer momento durante uma programação, sem a necessidade de redirecionamento para uma tela de autenticação, o que neste ultimo caso, ocasiona perda do conteúdo televisionado, ou a necessidade de redimensionamento da tela para que o $Q R$ Code possa ser disponibilizado ao lado da programação corrente. O QR Code ficará oculto durante toda a programação, aparecendo ao comando (de voz ou gestual) e sendo ocultado novamente após o comando referente a essa ação.

$\mathrm{Na}$ Figura 1 é possível ter uma visão esquemática do funcionamento da aplicação, onde se observa a interação dos usuários com a televisão e simultaneamente com o dispositivo de sensor de movimentos Kinect, escolhendo um dos modos de interação disponíveis, gesto ou voz e depois autenticando com um segundo dispositivo, ilustrado, neste caso, como um celular. Pode-se observar que os componentes envolvidos no funcionamento da aplicação encontram-se conectados à uma rede. 


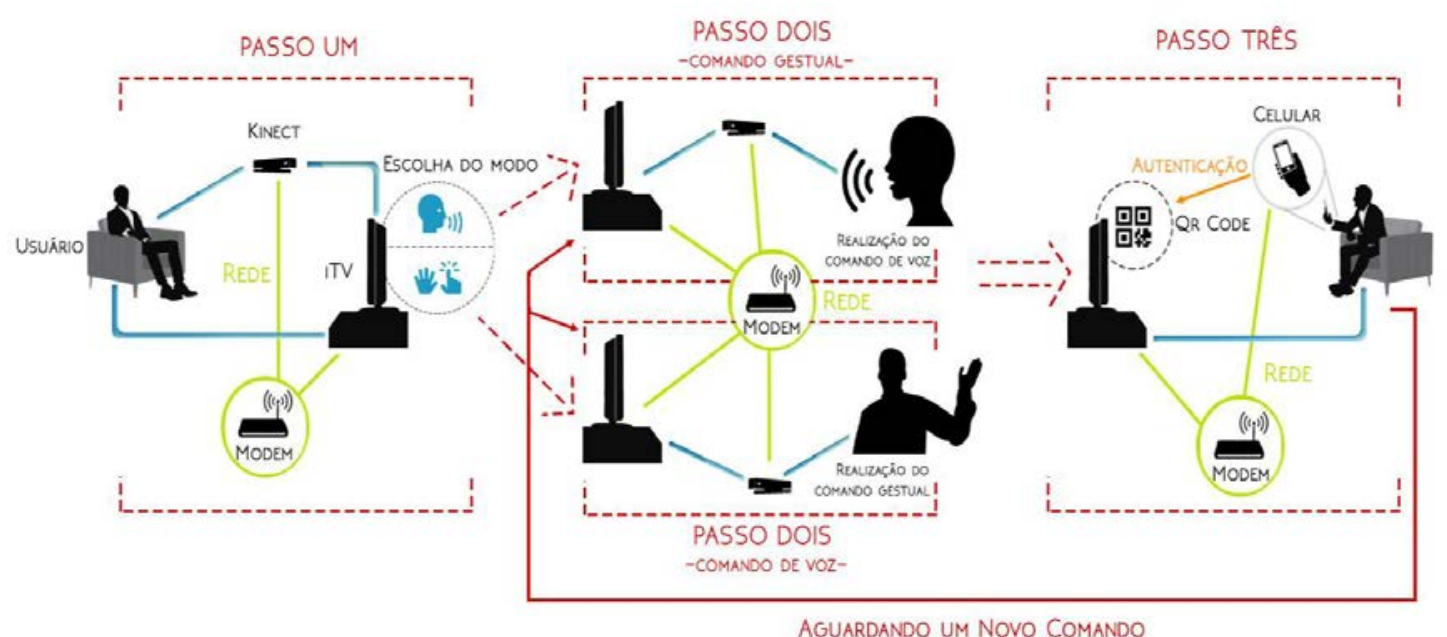

Figura 1- Visão Geral do Sistema

A aplicação foi desenvolvida através da IDE Microsoft Visual Studio ${ }^{1}$, com o auxílio do SDK do sensor de movimentos Kinect, periférico escolhido para a intermediação da interação entre usuário e aplicação, devido a sua relativa facilidade de manuseio e baixo custo de aquisição. O desenvolvimento da aplicação foi feito de forma a criar um módulo de fácil adaptação e acoplamento em distintas aplicações, tendo como foco inicial a inter-relação com aplicativos desenvolvidos em HTML5, para TV Digital.

O kinect apesar de ter sido criado inicialmente para a área de jogos passou a ser utilizado em diversas outras áreas como tecnologia de desenvolvimento de interfaces naturais, devido ao baixo custo e a grande capacidade computacional. [Silva 2013].

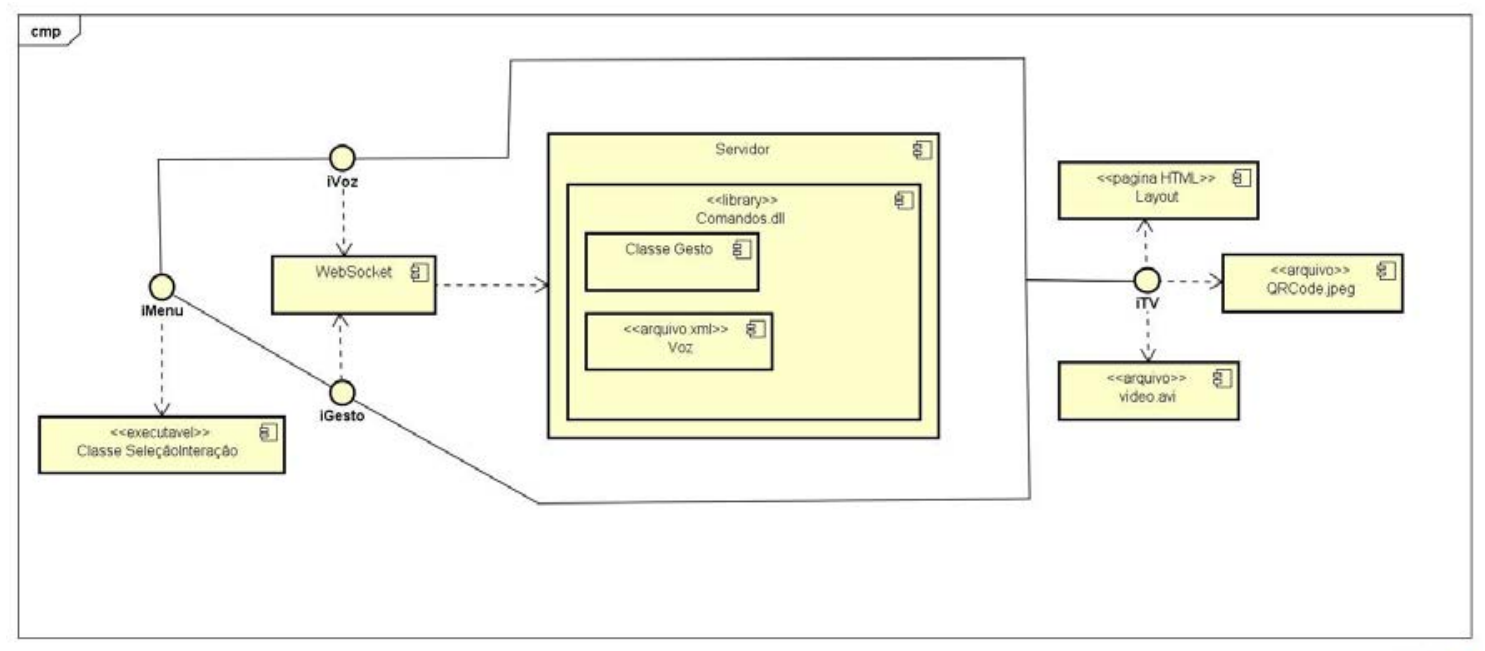

Figura 2 - Diagrama de Componentes do Sistema

A arquitetura da aplicação é demonstrada na Figura 2 através de um diagrama de componentes. È inicializada pela interface do menu de seleção do modo de interação desejado pelo usuário, que dependendo desta escolha é direcionado para a respectiva interface de cada interação natural, (voz ou gesto). A comunicação entre a aplicação da TV e o servidor é realizada através do websocket, no servidor está armazenada uma

\footnotetext{
${ }^{1}$ https://www.visualstudio.com
} 
biblioteca que contém os comandos gestuais e as palavras reservadas para realização da interação por voz. Outra interface do sistema é a da TVDi, que é composta por um arquivo HTML que contém um determinado vídeo (programação).

$\mathrm{Na}$ escolha da interação por gestos o servidor ficará aguardando novos comandos que serão enviados a partir do reconhecimento de um movimento especificado através do sensor de movimentos, enquanto a programação pode ser exibida normalmente, o gesto será identificado, enviando uma mensagem para o servidor C\#, através de websocket. O websocket retornará uma mensagem para a TV que então exibirá o $Q R$ Code.

Caso o usuário escolha a opção por comandos de voz o servidor ficará aguardando novos comandos que serão enviados a partir do reconhecimento da pronuncia de uma palavra especificada, enviando uma mensagem para o servidor $\mathrm{C} \#$, através de websocket. Novamente, o websocket retornará uma mensagem para a TV que então exibirá o $Q R$ Code, possibilitando ao usuário acessar os conteúdos adicionais através de seu dispositivo móvel.

Abaixo, na Figura 3 é apresentado o fluxo de execução em camadas de acesso, onde pode-se observar na camada superior, mais externa, as interfaces inicial, de gestos e de voz.

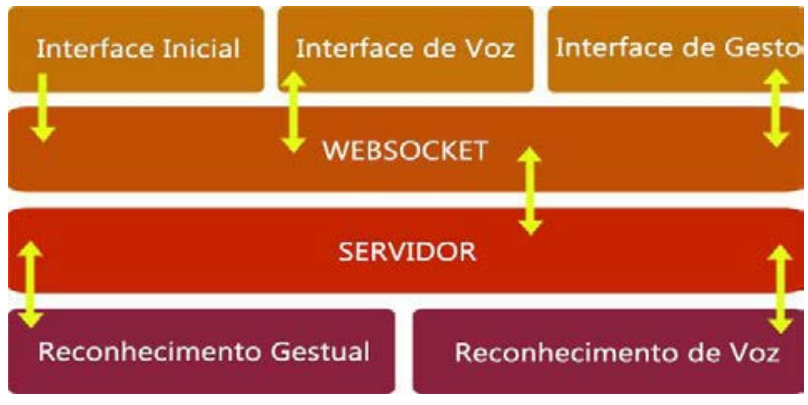

Figura 3. Fluxo de Execução em Camadas

Como demonstrado na Figura 3, a interface inicial é o ponto de partida. O fluxo segue então para a segunda camada, do websocket, onde poderá ser direcionado para uma das outras interfaces, podendo retornar a camada do websocket posteriormente e então passar para as camadas seguintes do servidor e finalmente a camada responsável pela identificação do comando realizado, retornando posteriormente por todas as camadas e exibindo a devida resposta a requisição na interface selecionada.

A proposta inicial era utilizar ambas as formas de interação na tela da interface inicial, porém, não foi possível realizar o preterido, devido à dificuldade de implementação dos dois modos de interação simultaneamente em uma interface, encontrada durante o desenvolvimento do aplicativo.

Parte da lógica das interfaces de gesto e voz é apresentada na Tabela 1, a seguir. Para a interação por voz, foram selecionadas três palavras base, sendo estas, "Play" para iniciar o vídeo da aplicação, "Show" para exibir o QR Code e "Hide" para ocultar.

Para interação natural por gestos o SDK do kinect disponibiliza as Joints, que são as articulações que o kinect consegue rastrear. Através da função de rastreamento do corpo é possível implementar diversos tipos de movimentos e poses utilizando Joints. $\mathrm{Na}$ Tabela 1 é demonstrado o uso das Joints (handRight e head). Na interface por gesto a pose da mão direita acima da cabeça foi implementada para ativar e desativar o $Q R$ 
Code, podendo-se observar, de forma sucinta, ambas as formas de implementação das interfaces naturais utilizando o kinect.

Tabela 1.Código das Interface de Voz e Gesto

\begin{tabular}{|c|c|}
\hline VOZ & GESTO \\
\hline $\begin{array}{l}\text { private void SpeechRecognized(object sender, } \\
\text { SpeechRecognizerEventArgs e) } \\
\text { //Iniciar video } \\
\text { const string Play = "PLAY"; } \\
\text { //Remover QRcode } \\
\text { const string Hide = "HIDE"; } \\
\text { //Habilitar QRcode } \\
\text { const string Show = "SHOW"; }\end{array}$ & $\begin{array}{l}\text { public class PoseQr : Pose }\{ \\
\text { public PoseQr() }\{ \\
\text { this. Nome = "PoseQr"; } \\
\} \\
\text { protected override bool ValidamentoPos(Skeleton } \\
\text { esqueletoUsuario) }\{ \\
\text { Joint maoDireita = } \\
\text { esqueletoUsuario.Joints[JointType.HandRight]; } \\
\text { Joint cabeca = } \\
\text { esqueletoUsuario.Joints[JointType.Head]; } \\
\text { Bool maoDireitaAlturaCorreta = } \\
\text { maoDireita.Position.Y > cabeca.Position.Y; } \\
\text { return maoDireitaAlturaCorreta; }\}\end{array}$ \\
\hline
\end{tabular}

Ambas as implementações foram destacadas para dar ênfase no processo de criação de interfaces multimodal. Para interação por voz, utilizou-se o método que disponibiliza serviços de comandos de voz SpeechRecognizer, para criação e configuração da gramática utilizada no arquivo XML que contém as palavras bases para utilização da aplicação.

$\mathrm{Na}$ codificação da interface por comandos gestuais, a classe "PoseQr" que habilita e desabilita o $Q R$ Code, herda parâmetros de validação e correção da classe "Pose", em caso da pose não estar completamente de acordo com o que foi préestabelecido para execução correta. O método "ValidamentoPos" rastreia as joints que estão sendo utilizadas, a variável "maoDireitaAlturaCorreta" verifica se a pose está sendo executada de forma correta, neste caso comparando a posição de ambas joints no eixo "Y" (vertical).

\section{Estudo de Caso}

Foram realizados testes com 26 usuários, selecionados aleatoriamente, entre alunos e professores dos diferentes cursos de graduação da Universidade Federal do Pará, campus Castanhal. A interatividade foi testada com mais de um usuário simultaneamente, desta forma, o aplicativo funciona a partir da interação natural apenas de um dos presentes usuários, então todos que estiverem assistindo a televisão podem interagir através da autenticação por $Q R$ Code com o conteúdo da segunda tela.

Para a realização dos testes procurou-se desenvolver uma atmosfera que simulasse o ambiente de utilização natural da televisão, tendo como base uma sala de estar, onde, buscando minimizar possíveis interferências e impactos negativos do ambiente de testes na interação do usuário com a televisão e o aplicativo, bem como obter resultados mais próximos possíveis do real, que poderiam ser observados em condições usuais.

Foram utilizados um sensor Kinect, para a detecção dos movimentos dos usuários, uma iTV onde foi disponibilizado o conteúdo de primeira tela, e um aparelho celular para a disponibilização do conteúdo de segunda tela, que consistia em conteúdo adicionais ao conteúdo exibido na primeira tela. 
Os testes deram-se segundo uma ordem preestabelecida, com o intuito de guiar os pesquisadores envolvidos e manter o controle sobre a realização dos testes e a coleta de dados de forma sistemática. Em uma etapa prévia, os usuários eram convidados a preencher um questionário de teor socioeconômico, com campos de informações básicas. Em seguida os usuários eram direcionados, individualmente, à sala de testes, com dois desenvolvedores e uma câmera, onde recebiam as instruções básicas necessárias para a realização dos testes.

Cada usuário realizou o teste na interface por comando de voz e na interface por comandos gestuais. O usuário tinha total liberdade para escolher a interface de interação inicial. Toda a aplicação era igual para as diferentes modalidades. É possível observar a utilização das distintas interfaces, de gesto e de voz respectivamente, nas Figuras 4.1 e 4.2, abaixo.

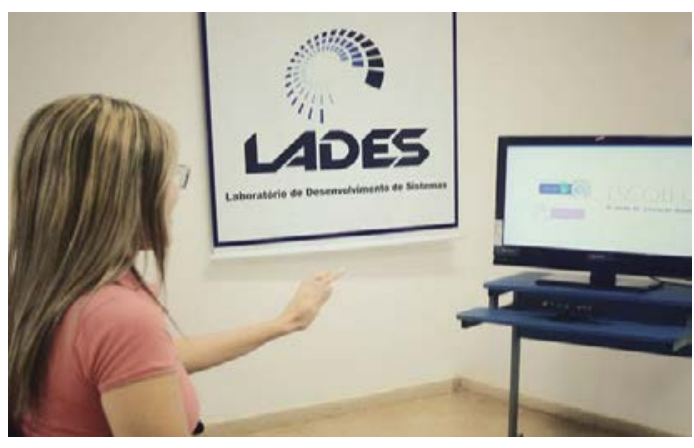

Figura 45.1 Realização dos TestesComando por Gesto

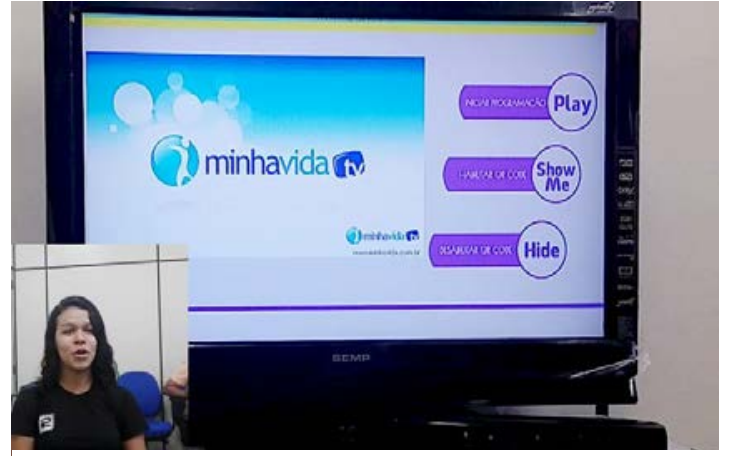

Figura 4.2 Realização dos TestesComando por Voz

Inicialmente era exibida uma tela com a mensagem indicando ao usuário que opta-se por um dos modos de interação indicados em dois botões. A escolha ocorre através de comando gestual. Na tela da interface escolhida eram disponibilizadas as opções relacionadas a cada interface de interação, botões para comandos gestuais e gestos para interface gestual e as palavras reservadas para interface por voz.

$\mathrm{Na}$ interface de comando gestual eram exibidos dois botões, um para iniciar ou pausar o vídeo disponibilizado e outro para exibir ou ocultar o $Q R$ Code, sendo que o usuário poderia, também, exibir e ocultar o $Q R$ Code através de uma pose previamente estabelecida na aplicação. Na interface de comando por voz eram exibidas, ao lado do vídeo, as palavras preestabelecidas, responsáveis por iniciar o vídeo, exibir e ocultar o QR Code, respectivamente, Play, Show e Hide.

Após exibir o $Q R$ Code os usuários podiam realizar o pareamento com o aparelho de segunda tela disponibilizado ao início do teste e utilizar livremente o conteúdo extra, disponibilizado na segunda tela. Após o fim do teste, os usuários preencheram formulários relacionados a qualidade de interação, utilização e nível de conhecimento prévio acerca das tecnologias utilizadas, através dos quais foram obtidos os dados necessários para a realização dos estudos, análises e delineamento dos perfis dos usuários.

Além dos questionários, também foram realizadas observações por parte dos pesquisadores, relacionadas ao comportamento desempenhado pelos usuários no decorrer dos testes. 


\section{Resultados}

Através da realização dos testes buscou-se definir padrões através da análise dos perfis de usuários, identificar o nível de satisfação dos usuários com relação à aplicação desenvolvida e detectar possíveis falhas e pontos a serem melhorados na mesma, desta forma disponibilizando este arcabouço de informações, novos desenvolvimentos que contemplam a mesma seara deste trabalho podem utilizar esta referência.

Os dados recolhidos através dos questionários foram tabulados para posterior analise. O perfil dos usuários da amostra dos 26 usuários convidados pode ser traçado, através da observação dos dados coletados, da seguinte forma na Figura 5: gênero dos usuários, a faixa etária, a renda mensal, o conhecimento prévio sobre NUI e $Q R$ Code.

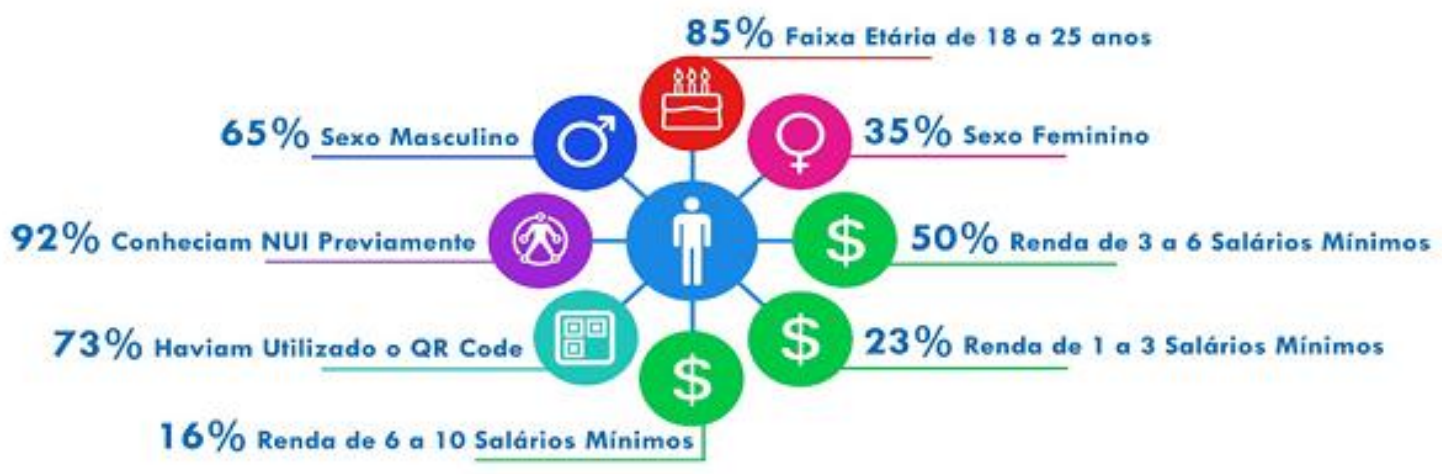

Figura 5. Perfil do Usuário

Como mostrado na Figura 5, 73\% dos usuários já haviam utilizado o $Q R$ Code anteriormente, a grande maioria para utilização do software whatsapp no computador, dessa forma apenas $27 \%$ não conhecia a tecnologia, desses mais da metade possuía renda de 1 a 3 salários mínimos. A tecnologia do $Q R$ Code, como mencionado anteriormente, foi utilizada para a autenticação e posterior utilização da segunda tela. A relação entre a renda dos usuários e o conhecimento prévio do $Q R$ Code é apresentada na Figura 6.

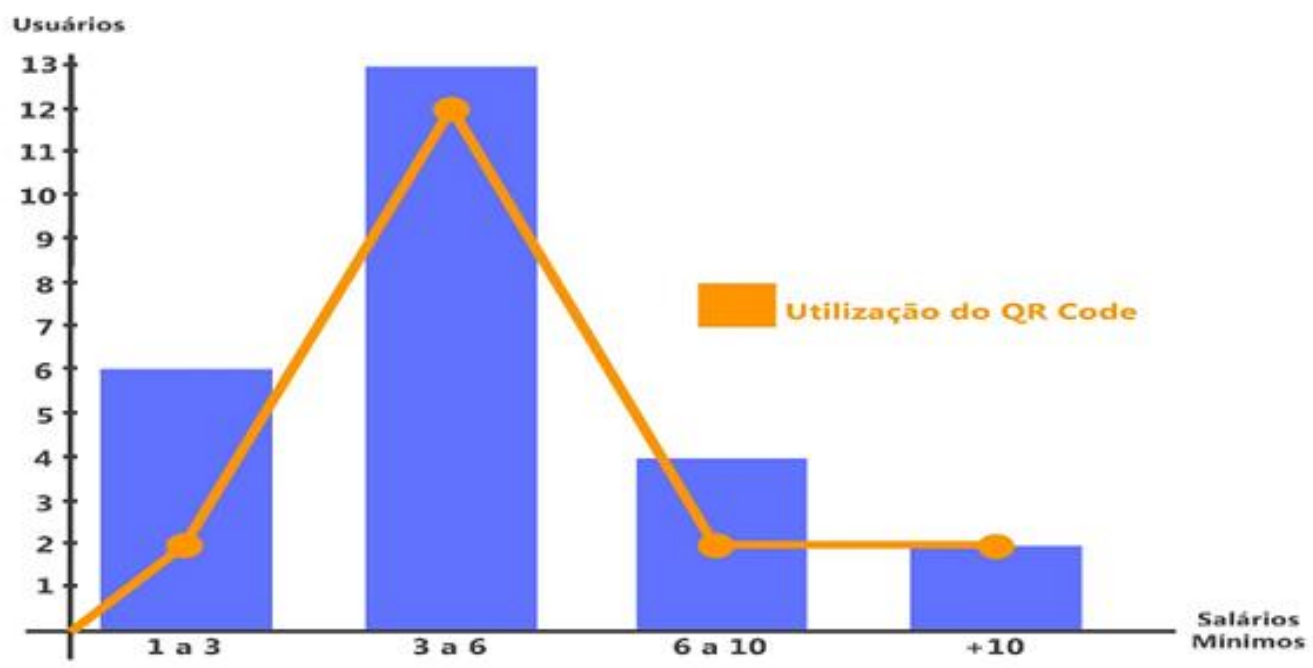

Figura 6. Relação entre nível de renda e conhecimento prévio sobre o QRCode

Dentre os participantes do teste, apenas $8 \%$, nunca haviam utilizado nenhum tipo de interface natural, fato que não afetou o desempenho dos usuários durante a 
interação com o sistema, embora a faixa de tempo utilizada na interação por gesto tenha sido relativamente alta, ela não fugiu ao padrão dos usuários com experiência prévia, da mesma forma com a interação por voz, obtendo, no entanto um tempo inferior à interação por gesto. Na Figura 7 é possível observar o tempo de execução para cada modo de interação.

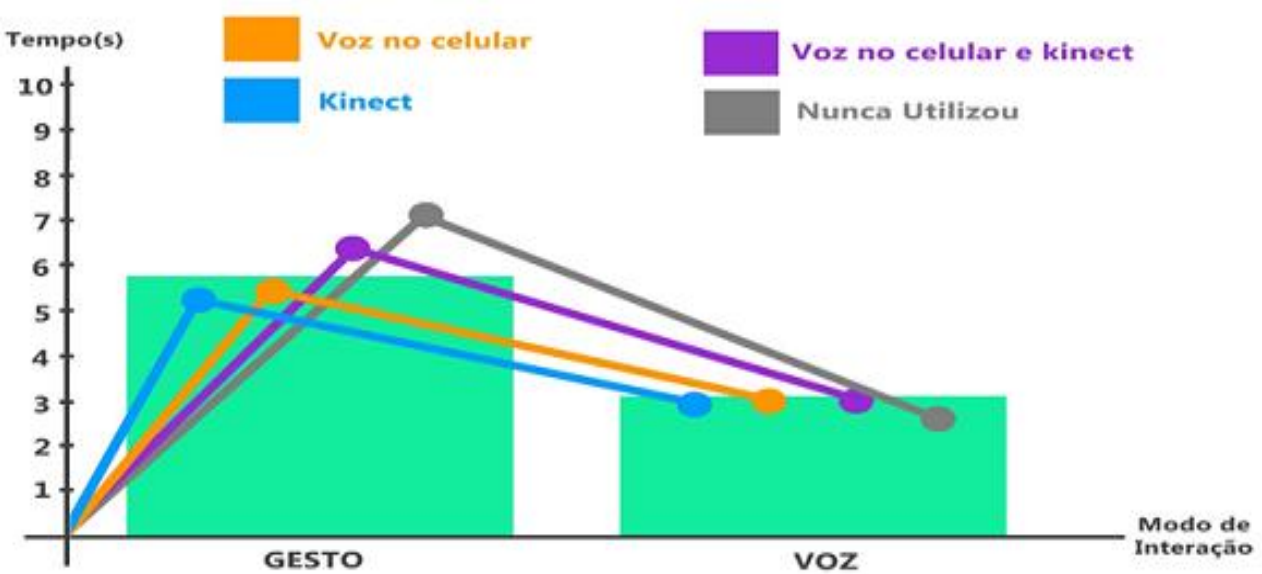

Figura 7. Tempo médio de execução para cada modo de interação e de cada segmento baseado na utilização prévia de NUI.

Os usuários levaram entre 5 e 6 segundos, para executar os comandos gestuais, enquanto que para efetuar os mesmos comandos por voz levaram cerca de 2 a 3 segundos, menos da metade do tempo que o primeiro como pode ser observado na Figura 8. Tal configuração era esperada, já que falar é muito mais intuitivo que fazer determinados gestos e poses predefinidas, porém, como mencionado anteriormente a escolha da pose pode, também, ajudar no desempenho do usuário, com base nisso a pose padrão foi substituída após a presente análise.

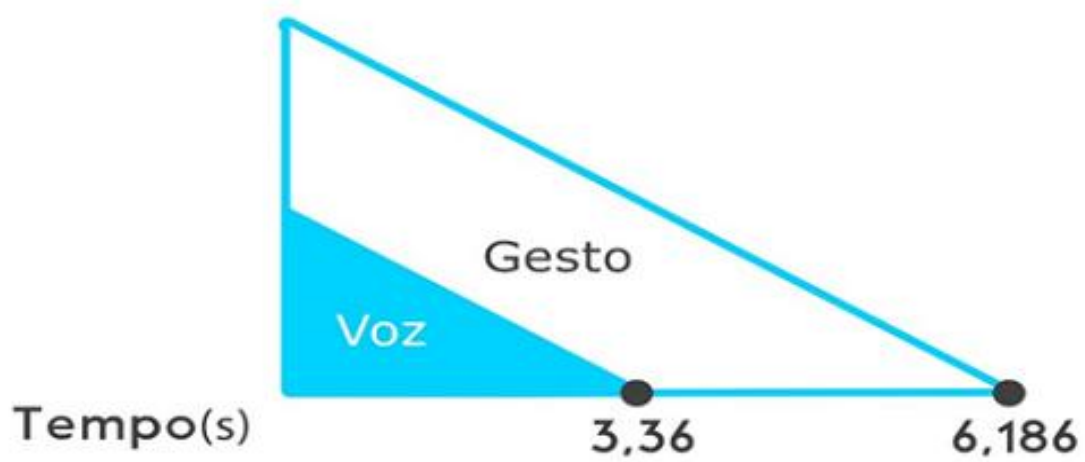

Figura 8. Área do tempo para primeira execução

Apesar de muito mais intuitiva a interface por comando de voz, teve como empecilho, durante a sua utilização, o fato das 3 palavras selecionadas serem em inglês, desta forma, o número de tentativas realizadas para que cada uma dos comandos predefinidos fossem executados com sucesso foi divergente entre eles (Figura 9), onde o comando utilizado com um maior grau de facilidade, ou seja, executado com um menor número de tentativas, foi o comando Show Me, seguido do comando Play, sendo que o comando de maior dificuldade de pronúncia e dessa forma de execução foi o comando Hide. 


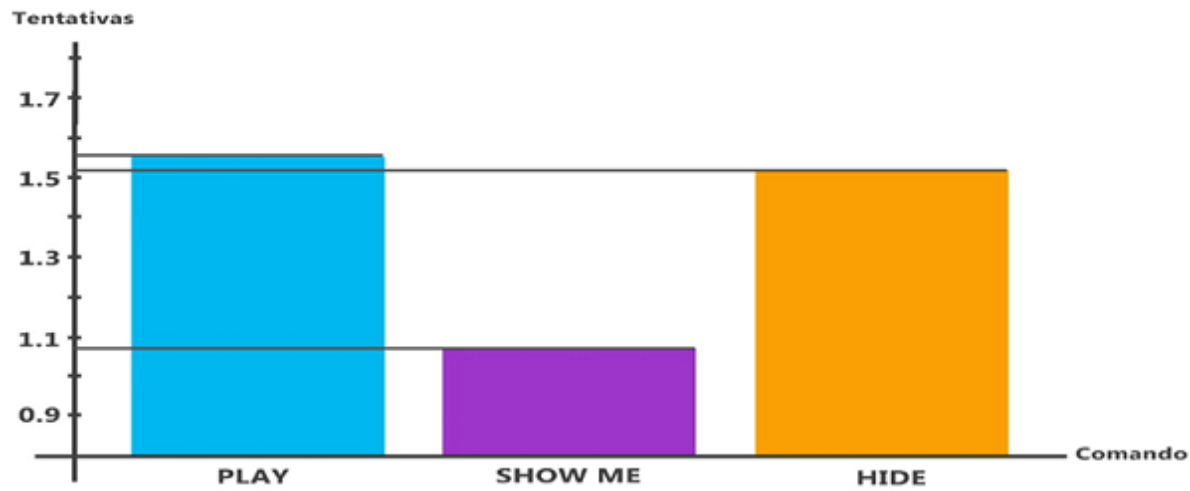

Figura 9. Média da variação do número de tentativas por comando

Um aspecto interessante relacionado à preferência dos usuários, com relação ao modo de interação, foi o fato de que todos os usuários que afirmaram preferir a interação por gesto, ainda não haviam utilizado essa tecnologia, os usuários que haviam utilizado interação gestual anteriormente além disso, foram unanimes ao declarar preferência pelo modo de interação por voz. (Figura 10)

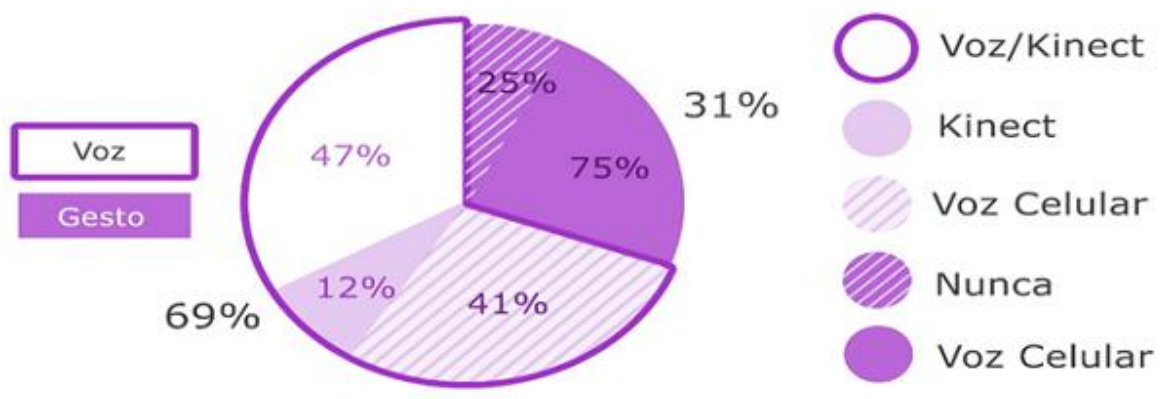

Figura 10. Preferência por interação relacionada ao uso anterior de NUI

De forma geral, os usuários não tiveram dificuldades em utilizar o aplicativo, mesmo os participantes que nunca haviam tido contato com Interfaces Naturais. $\mathrm{O}$ esforço e o tempo demandado para a execução do comando de voz foi inferior ao demandado para a execução dos comandos gestuais. O comando gestual mostrou-se bem aceito em um primeiro momento, porém, através da análise dos dados pode-se observar que quem já utilizou esse tipo de comando mais de uma vez tende a preferir o comando de voz por ser mais cômodo e eficaz.

\section{Conclusão}

O presente artigo expôs uma proposta utilizando aplicativo para TV Digital e Segunda Tela, para teste com interfaces como meios de interação, buscando dar ao usuário maior conforto, comodidade, e maior desempenho na utilização da TV Digital e da Segunda Tela.

A aplicação desenvolvida apesar de simples proporciona uma experiência nova para os telespectadores, e o aumento na qualidade de experiência do usuário, dando a este a possibilidade de utilizar sua TV segundo sua vontade, podendo operar ou não os recursos disponíveis.

Este trabalho permitiu a realização de uma análise distinta de interatividade do usuário, através de interfaces naturais para interação com a TV Digital e segunda tela, 
possibilitando a utilização de aplicações de interações híbridas, configurando um novo nível de interação. E os resultados obtidos nos testes, podem ser utilizados para comparação com outras pesquisas na área de NUI e IHC. Ressalta-se que a aplicação de segunda tela utilizada neste trabalho foi desenvolvida pelo próprio grupo de estudo do Laboratório de Desenvolvimento de Sistemas (LADES) da UFPA.

No intuito de aprimorar o estudo desenvolvido, pretende-se estabelecer uma comparação relacionada ao desempenho de diferentes dispositivos de NUI, como leap motion, levando em consideração a qualidade de experiência dos usuários associada a utilização dos diferentes dispositivos e modos de entrada.

\section{Referências}

Bobeth, J. Schrammel, S. Deutsch, M. Klein, M. Drobics, C. Hochleitner, and M. Tscheligi, Tablet, gestures, remote control?: influence of age on performance and user experience with iTV applications. In: Proc. of the 2014 ACM international conference on Interactive experiences for TV and online video, 139-146.

Cruickshank, L., Tsekleves, E., Whitham, R., Hill, A. 2007. Making interactive TV easier to use: interface design for a second screen approach. The Design Journal, 10 (3).

EY. Future of Television. EYGM Limited. 2013. Disponível em: http://www.ey.com/Publication/vwLUAssets/EY -

6 _trends_that_will_change_the_TV_industry/\$FILE/EY-6-trends-that-will-changethe-TV-industry.pdf

Fernandes, Jorge; Lemos, Guido; Silveira, Gledson. Introdução à televisão digital interativa: arquitetura, protocolos, padrões e práticas. In: Jornada de Atualização em Informática do Congresso da Sociedade Brasileira de Computação, 2004, Salvador. Anais do JAI-SBC. 56p.

Gazolla neto, Alexandre, et al. 2012. Rastreabilidade aplicada à produção de sementes de soja. Informativo ABRATES. v.22, n.2

IBOPE， DISPONÍVEL EM: < http://agenciafosforo.com.br/2014/03/midiasvidabrasileiros-pesquisa-ibope-2014/ >, Acessado em: 16 DE FEVEREIRO, 2015

Karray, F., M. Alemzadeh, J.A. Saleh, and M.N. Arab. Human-Computer Interaction: Overview on State of the Art. In International Journal on Smart Sensing and Intelligent Systems, Vol. 1, No. 1, March 2008.

Kong, J., W. Y. Zhang, N. Yu , X. J. Xia. Design of human-centric adaptive multimodal interfaces, International Journal of Human-Computer Studies, v.69 n.12, p.854-869, December, 2011.

SET. 2013 DISPONÍVEL EM: http://www.set.org.br/artigos/ed133/ed133_pag84.asp, acessado em 07 de abril, 2015

Silva, Renato K. Interfaces Naturais e o Reconhecimento das Línguas de Sinais. 2013. 130 p. Dissertação (Mestrado)-Pontíficia Universidade Católica de São Paulo, 2013

Tebbutt, David. In touch with tomorrow. In PC PRO, Feb 1995, p. 206-11. Dennis Publication, United Kingdom.

Turk, M. Multimodal interaction: A review. Pattern Recognition Lett. (2013), http://dx.doi.org/10.1016/ j.patrec.2013.07.003.

Valli, Alessandro. Notes on Natural Interaction. 2007. 